\title{
Study of LFS inorganic scintillator for applying to PET with 1x1 $\mathrm{mm}^{2}$ MPPC
}

\section{Makoto Yamazaki ${ }^{1}$}

The Division of Science of Technology, Graduate School of Shinshu University

3-1-1 Asahi Matsumoto city Nagano pref., 390-0861,Japan

E-mail: yamazaki@azusa.shinshu-u.ac.jp

\section{Tohru Takeshita ${ }^{2}$}

The Department of Science, Faculty of Science, Shinshu University

3-1-1 Asahi Matsumoto city Nagano pref., 390-0861,Japan

E-mail:tohru@shinshu-u.ac.jp

The main challenges in the design of next generation Positron Emission Tomography machines are the improvement of the spatial resolution and the combined operation with magnetic resonance. The Multi Pixel Photon Counter by Hamamatsu is a good candidate for this application, especially referring to newly developed Silicon photo sensor. Its small size $(1 \times 1$ $\mathrm{mm}^{2}$ ) and high photo-detection efficiency in the blue spectral region allow the direct readout of a highly segmented scintillator matrix with improvements on the detector's spatial resolution. Furthermore, this photodetector is insensitive to magnetic fields up to $5 \mathrm{~T}$, making its application in a magnetic resonance environment possible. The aim of the study presented is the characterization of a system of scintillator crystal readout via MPPC. Crystal of $3 \times 3 \times 15 \mathrm{~mm}^{3}$ are directly coupled to a MPPC, and the energy resolution at $511 \mathrm{keV}$ is measured to be $12 \% / \sqrt{E}$. It's sufficient to realize the PET. In addition, we measured the characters of LFS Lutetium Fine Silicate) compared with present general scintillators such as CsI or BGO. In this experiment, we got the enough number of photons by LFS which was attached to MPPC. The spatial resolution at $9 \mathrm{~cm}$ from the source point to be $1.4 \mathrm{~mm}$ is found.

International Workshop on New Photon Detectors (PD09)

Shinshu University Matsumoto Japan

24-26 June 2009

\footnotetext{
${ }^{1}$ Speaker
} 


\section{Introduction}

In recent days, medical imaging field is remarkably developing, especially on Positron Emission Tomography (PET). Beta+ decay RI is used and injected to inside of a human body with saccharide like FluoroDeoxy Glucouce (FDG). They get in the active cancer cells and decay into two gamma rays in back to back directions. Those gamma rays are detected by scintillators surrounding the body as $0.51 \mathrm{MeV}$ photoelectric peak of energy. Of course, two annihilation gamma rays are for any directions, so lay those lines up on the other then you can specify the interaction point where a cancer is. However, when it comes to edge of FOV (Field Of View), we will see parallax because of scintillator's own size. Therefore, to decrease parallax influence and get better picture, DOI (Depth of interaction) capability is suggested for now. Using this idea we can get good spatial resolution and fine view.

We used LFS (Lutetium Fine Silicate) inorganic scintillator which is made by Zecotek in Canada (Fig.1). It has high stopping power, therefore if it is possible to minimize its size to reduce parallax we could obtain a better special resolution. Furthermore it has short decay time and cheaper than LSO which is usually used in existent PET device. This crystal emits blue light ( $415 \mathrm{~nm})$, and MPPC is also sensitive to blue light region $(\sim 420 \mathrm{~nm})$, therefore it is ideal to combine them.

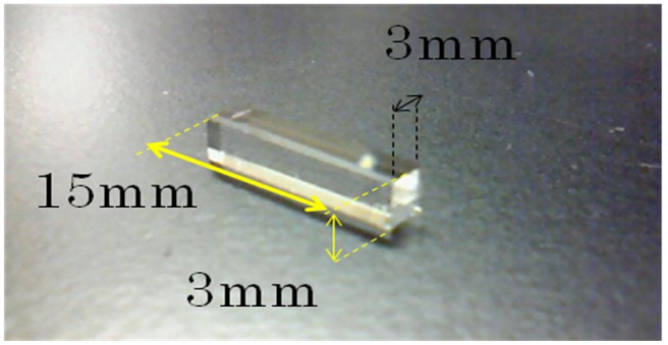

Fig.1 LFS (Lutetium Fine Silicate) manufactured by Zecotec in Canada. This sample has the size of $3 \times 3 \times 15 \mathrm{~mm}^{3}$. The surface is polished.

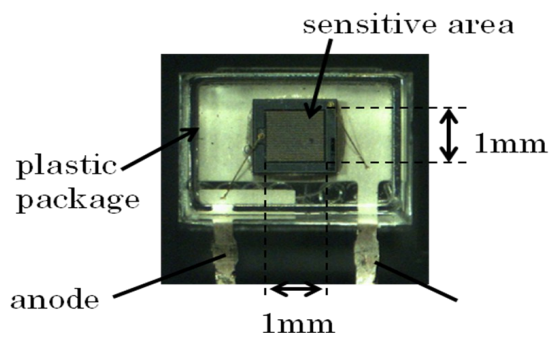

Fig.2 MPPC by HAMAMATSU in Japan. Sensitive area is the center of $3 \times 2.5 \mathrm{~mm}^{2}$ plastic package. This has 1600 pixels.

MPPC which is manufactured by HAMAMATSU in Japan is pixellized avalanche photodiode and has 1600 pixels in $1 \times 1 \mathrm{~mm}^{2}$ sensitive area (Fig.2). Each pixel size is $25 \mu \mathrm{m}$. Since each pixel outputs a signal for only one photon, the number of photo-electrons can be counted as the number of hit pixels. In addition, it can be operated at low bias $(\sim 76 \mathrm{~V})$ but it provide high gain $\left(10^{5} \sim 10^{6}\right)$. It's not less than classical PMT and really enough to be alternative of it. 


\section{Setup outline}

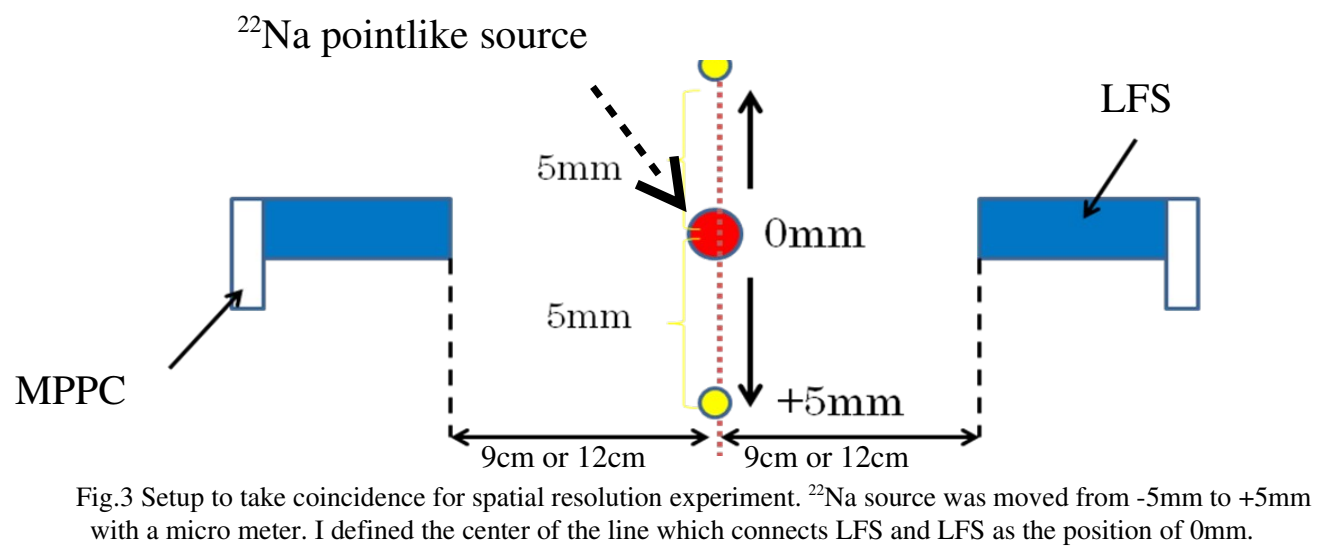

\subsection{Experimental setup}

LFS and MPPC were coupled with each other directly without any optical glue. Then it was connected to readout circuit.

We used two pairs of MPPC and LFS combination detector to take coincidence of annihilation gamma rays. They were placed along a line, and fixed face to face with each other. Readout signals from MPPC were input into discriminator (N023, HOSIN) at first without any amplification, then logic output signal was sent into coincidence module (N013, HOSIN). One output signal of the coincidence module was used as a gate signal of ADC module (C009 16CH ADC, HOSHIN), and the other was fed into scalar (KN1860, KAIZU) to count coincidence for studying spatial resolution. To take energy distribution the output signal of a MPPC was fed into ADC. The RI source was ${ }^{22} \mathrm{Na}$ which emits $0.51 \mathrm{MeV}$ and $1.28 \mathrm{MeV}$ gamma rays. It was placed at the midpoint between two LFSs (Fig.3). Radiation from the source was not collimated since this setup should be close to real examination condition in which we cannot collimate the annihilation gamma rays from RI injected inside of body.

\subsection{Energy resolution}

In this time, distance between LFS to LFS is set to $9 \mathrm{~cm}$. In addition to annihilation gamma rays ${ }^{22} \mathrm{Na}$ emits $1.28 \mathrm{MeV}$ gamma rays simultaneously which induce background signals in this experimental setup assumed as the PET system. Therefore, by taking coincidence, we have to discriminate gamma rays from the annihilation from the background.

\subsection{Spatial resolution}

We counted the number of coincidence from two LFS's by changing the position of source set on the stage driven by a micrometer by $1 \mathrm{~mm}(-5 \mathrm{~mm} \sim+5 \mathrm{~mm})$ along the line perpendicular 
to the one which connects two LFSs (Fig.3). In addition, in a different condition where the distance between LFSs was changed to $12 \mathrm{~cm}$, the same measurement was performed. To construct a new PET device we need millimeter order resolution, especially for micro-PET for the experiment using rodents, so it is important to know the achievable resolutions of the system.

\section{Result}

\subsection{Energy resolution}

Fig. 4 shows ADC spectrum. Vertical axis represents the number of counts per minute, and horizontal axis is ADC channels. Photo-electric peak of $0.511 \mathrm{MeV}$ is shown and fitted in red gaussian curve. We obtained energy resolution $\sigma(\mathrm{E}) / \mathrm{E}$ of $11.8 \pm 0.1 \%$.

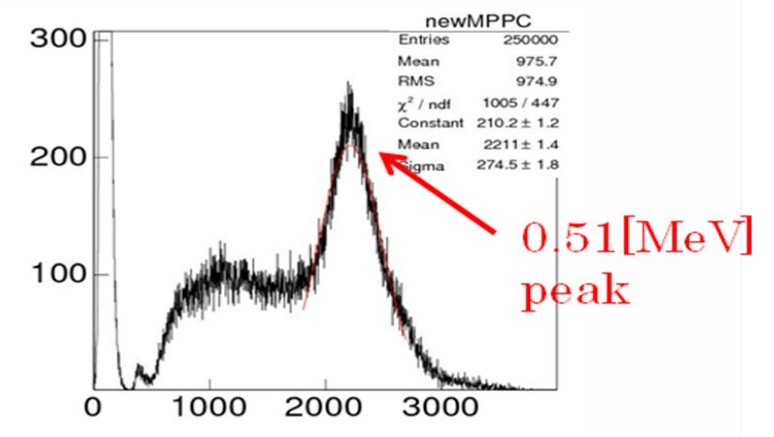

Fig.4 Energy distribution of $0.51 \mathrm{MeV}$ gamma ray from ${ }^{22} \mathrm{Na}$

\subsection{Spatial resolution}

Spatial resolution is tested on two conditions. First, distance between LFS and source was set to $9 \mathrm{~cm}$. This is the same condition as the energy resolution was measured. The dots and the solid line in Fig. 5 show the number of coincident events as the function of the source position and the fitting result of the Gauss function, respectively. The spatial resolution was $1.79 \pm 0.17$ $\mathrm{mm}$.

The same measurement was performed for the different setup where the distance between LFSs is $12 \mathrm{~cm}$ and spatial resolution was determined. In this setup, the spatial resolution of $\sigma=$ $1.38 \pm 0.05 \mathrm{~mm}$ was obtained as shown in Fig. 6 .

As shown in Fig.5 and Fig.6, we can see the offset of the number of counts around 1.52 times $10^{6}$. This may be because of accidental coincidence between $1.28 \mathrm{MeV}$ gamma rays and one of the annihilation gamma rays from ${ }^{22} \mathrm{Na}$. While detecting $0.51 \mathrm{MeV}$ annihilation gamma simultaneously, 1.28 MeV gamma is emitted in any directions. Therefore, it can be background. 
In case that distance from detector to source becomes larger, parallax is decreased and this results in better spatial resolution, while detection efficiency is declined according to $1 / \mathrm{r}^{2}$. $\mathrm{r}$ means here the distance between source and a LFS crystal. In addition, our purpose on this work is to construct micro PET for rodents, therefore we do not need large gantry making detection efficiency low, but good spatial resolution. To decide suitable size of gantry and spatial resolution for micro PET, further studies are needed.

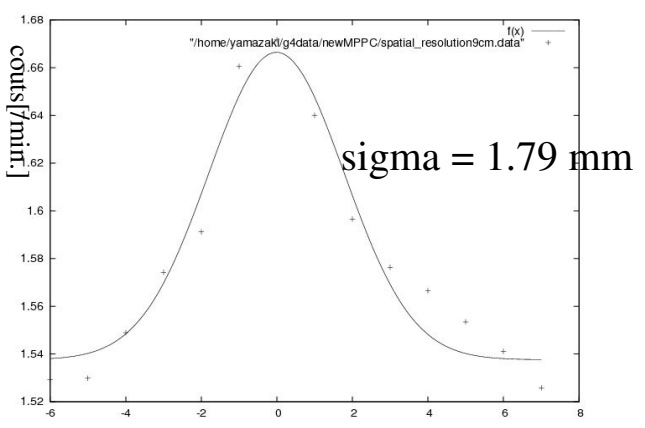

position[mm]
Fig.5 spatial resolution at $9 \mathrm{~cm}$ distance. Vertical axis shows the number of counts $\left(\mathrm{x} 10^{6}\right)$, and horizontal axis for position ([mm]).

Conclusion

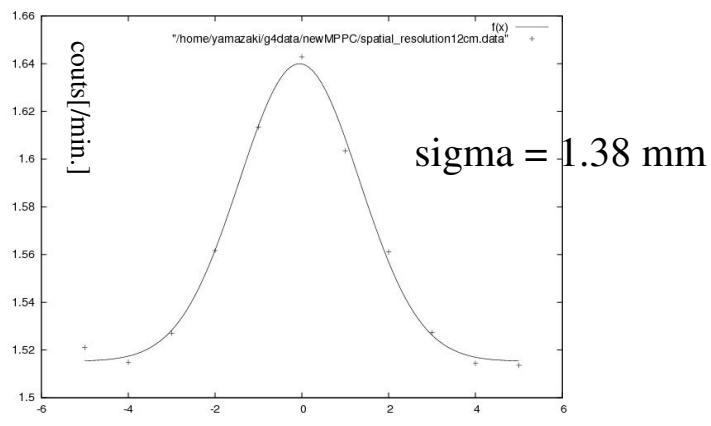

position[mm]

Fig. 6 spatial resolution at $12 \mathrm{~cm}$ distance

We measured the energy resolution and spatial resolution of the primitive PET system with LFS and MPPC on this work, and got promising results for applying to PET. Energy resolution is $12 \%$ and spatial resolution is $1.4 \mathrm{~mm}$ at sigma in the case of $12 \mathrm{~cm}$ distance from detector to source. We will measure with BGO and LSO crystals for comparison of output light and different signal shapes, and get more data at different distance, especially at farer one, and plot with suitable function. It will make the result clearer on relation of spatial resolution and distance. Then we will measure time resolution. LFS has really short decay time, so it could be possible to apply it to TOF (Time Of Flight) PET, if a nice time resolution can be obtained, we suggest another idea for new PET device.

\section{References}

N.D'Ascenzo, E.Garutti, A.Tadday, Application of MPPC to Positron Emission Tomography, POS (PD07) 006

http://pos.sissa.it/cgi-bin/reader/contribution.cgi?id=PoS (PD07) 006

[1] M.Gottlich, E.Garutti, V.Kozlov, Hans-Christian Schultz-Coulon, A.Tadday, A.Terkulov, Application of Multi-Pixel Photon Counter to Positron Emission Tomography, IEEE Trans. Nucl. Sci., 2008 